PROCEEDINGS OF THE

AMERICAN MATHEMATICAL SOCIETY

Volume 126, Number 2, February 1998, Pages 425-430

S $0002-9939(98) 04060-X$

\title{
ON THE ASYMPTOTICITY ASPECT OF HYERS-ULAM STABILITY OF MAPPINGS
}

\author{
D. H. HYERS, G. ISAC, AND TH. M. RASSIAS \\ (Communicated by Palle E. T. Jorgensen)
}

\begin{abstract}
The object of the present paper is to prove an asymptotic analogue of Th. M. Rassias' theorem obtained in 1978 for the Hyers-Ulam stability of mappings.
\end{abstract}

\section{INTRODUCTION}

In [15] Rassias generalized the result of Hyers [9] by allowing growth of the form $\varepsilon \cdot\left(\|x\|^{p}+\|y\|^{p}\right)$ for the norm of the Cauchy difference $f(x+y)-f(x)-f(y)$, where $0 \leq p<1$, and still obtained the formula

$$
g(x)=\lim _{n \rightarrow \infty} \frac{f\left(2^{n} x\right)}{2^{n}}
$$

for the additive mapping approximating $f$. Other developments of this idea are described in [10] (see also [1], [5], [7], [8], [12], [13], [16]). In the present article we obtain an asymptotic analogue of this result of Th. M. Rassias.

Several authors have used asymptotic conditions in stating approximations to Cauchy's functional equation

$$
f(x+y)=f(x)+f(y) .
$$

P.D.T.A. Elliott [6] showed that if the real function $f$ belongs to the class $L^{p}(0, z)$ for every $z \geq 0$, where $p \geq 1$, and satisfies the asymptotic condition

$$
\lim _{z \rightarrow \infty} \frac{\int_{0}^{z} \int_{0}^{z}|f(x+y)-f(x)-f(y)|^{p} d x d y}{z}=0,
$$

then there is a constant $c$ such that $f(x)=c x$ almost everywhere on $\mathbb{R}^{+}$. One of the theorems of J. R. Alexander, C. E. Blair and L. A. Rubel [1] states that if $f \in L^{1}(0, b)$ for all $b>0$, and if for almost all $x>0$

$$
\lim _{u \rightarrow \infty} \frac{\int_{0}^{y}[f(x+y)-f(x)-f(y)] d y}{u}=0,
$$

then for some real number $c, f(x)=c x$ for almost all $x \geq 0$.

Received by the editors December 11, 1995 and, in revised form, May 21, 1996 and July 29, 1996.

1991 Mathematics Subject Classification. Primary 39B72, 47H15.

Key words and phrases. Hyers-Ulam stability, asymptotic conditions, asymptotically derivable, additive outside a ball.

(C)1998 American Mathematical Society 
F. Skof [17] proved that given real normed spaces $X$ and $E$ and a mapping $f: X \rightarrow E$ satisfying the condition

$$
\|f(x+y)-f(x)-f(y)\| \rightarrow 0 \text { as }\|x\|+\|y\| \rightarrow \infty,
$$

then $f(x+y)=f(x)+f(y)$ for all $x$ and $y$ in $X$. In a later article [18] the same author showed that a real-valued function $f$ defined on a real normed space $X$ is additive proving that $f(0)=0$ and $|f(x+y)|-|f(x)+f(y)| \rightarrow 0$ when $\|x\|+\|y\| \rightarrow 0$. In [12] is shown an interesting relation between the Hyers-Ulam stability and the asymptotic derivability. This relation is applied to the study of some important nonlinear problems (cf. [13]).

In the present paper we consider the asymptoticity aspect of Hyers-Ulam stability close to the asymptotic derivability. The asymptotic derivability is very important in nonlinear analysis (cf. [2], [3], [4], [11], [14]).

\section{Main Result}

Theorem 1. Given a real normed vector space $E_{1}$ and a real Banach space $E_{2}$, let numbers $M>0, \varepsilon>0$ and $p$ with $0<p<1$ be chosen. Let the mapping $f: E_{1} \rightarrow E_{2}$ satisfy the inequality

$$
\|f(x+y)-f(x)-f(y)\| \leq \varepsilon\left(\|x\|^{p}+\|y\|^{p}\right)
$$

for all $x, y$ in $E_{1}$ such that

$$
\|x\|^{p}+\|y\|^{p}>M^{p}
$$

Then there exists an additive mapping $\varphi: E_{1} \rightarrow E_{2}$ such that

$$
\|\varphi(x)-f(x)\|<\beta(p) \varepsilon\|x\|^{p}
$$

for all $x \in E_{1}$ with $\|x\|>\frac{M}{2^{1 / p}}$, where $\beta(p)=\frac{2}{2-2^{p}}$ and $\varphi(x)=\lim _{n \rightarrow \infty} \frac{f\left(2^{n} x\right)}{2^{n}}$.

Proof. When $\|x\|>\frac{M}{2^{1 / p}}$, that is, when $2\|x\|^{p}>M^{p}$, we may put $y=x$ in (1) to obtain

$$
\left\|2^{-1} f(2 x)-f(x)\right\| \leq \varepsilon\|x\|^{p} .
$$

Of course we can replace $x$ by $2 x$ in (4) since $\|2 x\|$ is also greater than $\frac{M}{2^{1 / p}}$. Thus, we can use the argument given in [15] to arrive at the inequality

$$
\left\|2^{-n} f\left(2^{n} x\right)-f(x)\right\| \leq \beta(p) \varepsilon\|x\|^{p} \quad \text { when }\|x\|>\frac{M}{2^{1 / p}} \text { for } n \in N
$$

and thus to show that the limit

$$
g(x)=\lim _{n \rightarrow \infty} \frac{f\left(2^{n} x\right)}{2^{n}}
$$

exists when $\|x\|>\frac{M}{2^{1 / p}}$. Therefore

$$
\|g(x)-f(x)\| \leq \beta(p) \varepsilon\|x\|^{p} .
$$

Clearly, when $\|x\|>\frac{M}{2^{1 / p}}, g(2 x)=\lim _{n \rightarrow \infty} \frac{f\left(2^{n+1} x\right)}{2^{n}}=2 \lim _{n \rightarrow \infty} \frac{f\left(2^{n+1} x\right)}{2^{n+1}}$, so that

$$
g(2 x)=2 g(x) \quad \text { for }\|x\|>\frac{M}{2^{1 / p}} .
$$

Now suppose that $\|x\|,\|y\|$ and $\|x+y\|$ are all greater than $\frac{M}{2^{1 / p}}$. Then by (1) we find that for all $n \in N$,

$$
\left\|2^{-n} f\left(2^{n}(x+y)\right)-2^{-n} f\left(2^{n} x\right)-2^{-n} f\left(2^{n} y\right)\right\| \leq \varepsilon 2^{-n(1-p)}\left(\|x\|^{p}+\|y\|^{p}\right) .
$$


Under the conditions stated it follows by (6) that

$$
g(x+y)=g(x)+g(y) .
$$

Using an extension method of F. Skof [18] we will define a mapping $\varphi: E_{1} \rightarrow E_{2}$ to be an extension of the mapping $g$ to the whole space $E_{1}$. Given any $x \in E_{1}$ with $0<\|x\|<\frac{M}{2^{1 / p}}$, let $k=k(x)$ denote the largest integer such that

$$
\frac{M}{2^{1 / p}}<2^{k}\|x\| \leq M
$$

Define the mapping $\varphi$ as follows:

$$
\begin{cases}\varphi(0)=0, & \\ \varphi(x)=2^{-k} g\left(2^{k} x\right) & \text { for } 0<\|x\|<\frac{M}{2^{1 / p}}, \text { where } k=k(x), \\ \varphi(x)=g(x) & \text { for }\|x\|>\frac{M}{2^{1 / p}} .\end{cases}
$$

Lemma. For all $x$ in $E_{1}$

$$
\varphi(x)=\lim _{s \rightarrow \infty} 2^{-s} f\left(2^{s} x\right)
$$

and

$$
\varphi(-x)=-\varphi(x) .
$$

Proof. Take any $x$ in $E_{1}$ with $0<\|x\|<\frac{M}{2^{1 / p}}$, and let $k=k(x)$, so that $k$ is the largest integer satisfying (10). Thus $k-1$ is the largest integer satisfying

$$
\frac{M}{2^{1 / p}}<\left\|2^{k-1}(2 x)\right\| \leq M
$$

and we have

$$
\varphi(2 x)=2^{-(k-1)} g\left(2^{k-1}(2 x)\right)=2^{-k} \cdot 2 g\left(2^{k} x\right)=2 \varphi(x) \quad \text { for } 0<\|x\|<\frac{M}{2^{1 / p}} .
$$

From the definition of $\varphi$ and property (8) of $g$ it follows that $\varphi(2 x)=2 \varphi(x)$ for all $x$ in $E_{1}$. Given $x$ in $E_{1}$ with $x \neq 0$, choose a positive integer $m$ so large that $\left\|2^{m} x\right\|>\frac{M}{2^{1 / p}}$.

By the definition of $\varphi$ we have

$$
\varphi(x)=2^{-m} \varphi\left(2^{m} x\right)=2^{-m} g\left(2^{m} x\right),
$$

and by (6) this implies that

$$
\varphi(x)=\lim _{n \rightarrow \infty} 2^{-(m+n)} f\left(2^{m+n} x\right),
$$

which demonstrates (11) for $x \neq 0$.

Since $\varphi(0)=0$, the same is true for $x=0$. Equation (12) is obvious for $x=0$. Take any $x$ in $E_{1}$ with $x \neq 0$ and choose $n \in N$ large enough so that $\left\|2^{n} x\right\|>\frac{M}{2^{1 / p}}$.

Then by (1) with $y=-x$ we obtain

$$
\left\|2^{-n} f\left(2^{n} x\right)+2^{-n} f\left(-2^{n} x\right)\right\| \leq 2 \varepsilon 2^{-n(1-p)}\|x\|^{p}+2^{-n}\|f(0)\| .
$$

When $n \rightarrow \infty$ it follows from (11) that (12) holds. The lemma is proved.

In proving the additivity of $\varphi$ we note that the equation

$$
\varphi(x+y)=\varphi(x)+\varphi(y)
$$

holds when either $x$ or $y$ is zero.

Assume then that $x \neq 0$ and $y \neq 0$. If $x+y=0$, i.e. $y=-x$, then (12) shows that (13) holds. The only remaining case is when $x, y$ and $x+y$ are all different from 
zero. In this case we may choose an $n$ in $N$ such that $\left\|2^{n} x\right\|,\left\|2^{n} y\right\|$ and $\left\|2^{n}(x+y)\right\|$ are all greater than $\frac{M}{2^{1 / p}}$. Then by (1) we have

$$
\left\|f\left(2^{n}(x+y)\right)-f\left(2^{n} x\right)-f\left(2^{n} y\right)\right\| \leq \varepsilon 2^{n p}\left(\|x\|^{p}+\|y\|^{p}\right) .
$$

If we divide both sides of this inequality by $2^{n}$ and then let $n \rightarrow \infty$, we find by (11) that (13) is true, thus $\varphi$ is additive.

By definition $\varphi(x)=g(x)$ when $\|x\|>\frac{M}{2^{1 / p}}$, thus (3) follows from (7) and the proof of Theorem 1 is complete. Q.E.D. 1.

For convenience in applications we give the following modified version of Theorem

Theorem 2. Given a real normed vector space $E_{1}$ and a real Banach space $E_{2}$, let numbers $m>0, \varepsilon>0$ and $p$ with $0 \leq p<1$ be chosen. Suppose that the mapping $f: E_{1} \rightarrow E_{2}$ satisfies the inequality

$$
\|f(x+y)-f(x)-f(y)\| \leq \varepsilon\left(\|x\|^{p}+\|y\|^{p}\right)
$$

for all $x$ and $y$ in $E_{1}$ such that $\|x\|>m,\|y\|>m$ and $\|x+y\|>m$. Then there exists an additive mapping $\varphi: E_{1} \rightarrow E_{2}$ which satisfies

$$
\|\varphi(x)-f(x)\| \leq 2 \varepsilon\left(2-2^{p}\right)^{-1}\|x\|^{p}
$$

for all $x$ in $E_{1}$ such that $\|x\|>m$. Moreover, $\varphi$ is given by the formula

$$
\varphi(x)=\lim _{n \rightarrow \infty} 2^{-n} f\left(2^{n} x\right)
$$

for all $x$ in $E_{1}$.

Proof. Assume that $\|x\|>m$. Then as in the proof of Theorem 1 we obtain (4)-(8) inclusive, but now all these formulas are satisfied for $\|x\|>m$. In particular,

$$
g(x)=\lim _{n \rightarrow \infty} 2^{-n} f\left(2^{n} x\right) \quad \text { when }\|x\|>m .
$$

Also, if $\|x\|>m,\|y\|>m$ and $\|x+y\|>m$, then by hypothesis we see that (9) and (9a) also hold. To apply Skof's extension procedure in the present case, let $x$ in $E_{1}$ be given with $0<\|x\| \leq m$ and define $k=k(x)$ to be the unique positive integer such that

$$
m<2^{k}\|x\| \leq 2 m
$$

Now define the mapping $\varphi: E_{1} \rightarrow E_{2}$ as follows:

$$
\begin{cases}\varphi(0)=0, & \\ \varphi(x)=2^{-k} g\left(2^{k} x\right) & \text { for } 0<\|x\| \leq m, \\ \varphi(x)=g(x) & \text { for }\|x\|>m .\end{cases}
$$

The proof of the Lemma used in the proof of Theorem 1, follows as before with the obvious changes.

Indeed, we start with $x$ in $E_{1}$ satisfying $0<\|x\| \leq m$ and let $k=k(x)$ as defined by (14), etc. Thus the Lemma holds under the conditions of Theorem 2 . The proof of the additivity of $\varphi$ also follows as before. Therefore the proof of Theorem 2 is complete. Q.E.D. 


\section{3. p-ASYMPTOTICAL ADDITIVITY}

We apply the main theorem, precisely Theorem 2 , to the study of $p$-asymptotical derivatives.

Let $E_{1}$ and $E_{2}$ be Banach spaces. Let $T$ be a mapping from $E_{1}$ into $E_{2}$ satisfying eventually a special property such as, for example, additivity, linearity, etc. Let $0<p<1$ be arbitrary.

Definition 1. A mapping $f: E_{1} \rightarrow E_{2}$ is p-asymptotically close to $T$ if and only if $\lim _{\|x\| \rightarrow \infty} \frac{\|f(x)-T(x)\|}{\|x\|^{p}}=0$.

Remark 1. If in Definition $1, T \in L\left(E_{1}, E_{2}\right)$, then we say that $T$ is a $p$-asymptotical derivative of $f$ and if such a $T$ exists, then $f$ is p-asymptotically derivable.

Remark 2. Since for $x$ such that $\|x\| \geq 1$ we have $\|x\|^{p} \leq\|x\|$, one obtains that every $p$-asymptotical derivative of $f$ is an asymptotical derivative. Indeed, if $T \in$ $L\left(E_{1}, E_{2}\right)$ is a $p$-asymptotical derivative of $f$, then

$$
0 \leq \lim _{\substack{\|x\| \rightarrow \infty \\\|x\|>1}} \frac{\|f(x)-T(x)\|}{\|x\|} \leq \lim _{\substack{\|x\| \rightarrow \infty \\\|x\|>1}} \frac{\|f(x)-T(x)\|}{\|x\|^{p}}=0 .
$$

Definition 2. A mapping $f: E_{1} \rightarrow E_{2}$ is p-asymptotically additive if and only if for every $\varepsilon>0$ there exists $\delta>0$ such that

$$
\|f(x+y)-f(x)-f(y)\| \leq \varepsilon\left(\|x\|^{p}+\|y\|^{p}\right)
$$

for all $x, y \in E$ such that $\|x\|^{p},\|y\|^{p},\|x+y\|^{p}>\delta$.

Definition 3. A mapping $T: E_{1} \rightarrow E_{2}$ is additive outside a ball if there exists $r>0$, such that $T(x+y)=T(x)+T(y)$ for all $x, y \in E_{1}$ with $\|x\|,\|y\| \geq r$ and $\|x+y\| \geq r$.

Example. Let $T: E_{1} \rightarrow E_{2}$ be defined by

$$
T(x)= \begin{cases}L(x) & \text { if }\|x\| \geq r \\ \varphi(x) & \text { if }\|x\|<r\end{cases}
$$

where $L: E_{1} \rightarrow E_{2}$ is a linear mapping and

$$
\varphi: B(0, r) \rightarrow E_{2}
$$

is a nonlinear mapping where $B(0, r)=\left\{x \in E_{1} \mid\|x\|<r\right\}$. It follows that if $x, y \in E_{1}$ with $\|x\| \geq r,\|y\| \geq r$, and $\|x+y\| \geq r$, then $T(x+y)=T(x)+T(y)$.

We have the following result.

Theorem 3. If $f: E_{1} \rightarrow E_{2}$ is p-asymptotically close to an additive mapping outside a ball $T: E_{1} \rightarrow E_{2}$, then $f$ is p-asymptotically additive.

Theorem 4. If $f: E_{1} \rightarrow E_{2}$ is p-asymptotically close to an additive outside a ball mapping $T: E_{1} \rightarrow E_{2}$, then $f$ is $p_{*}$-asymptotically close to an additive mapping, where $0<p<p_{*}<1$.

Corollary. If $f: E_{1} \rightarrow E_{2}$ is p-asymptotically close to an additive outside a ball mapping $T: E_{1} \rightarrow E_{2}$, then $f$ has an additive asymptotical derivative.

\section{ACKNOWLEDGMENT}

We wish to express our thanks to the referees for their helpful remarks. 


\section{REFERENCES}

1. R. Alexander, C. E. Blair and L. A. Rubel, Approximate version of Cauchy's functional equation, Illinois J. Math. 39 (1995), 278-287. MR 96b:39018

2. H. Amann, Fixed points of asymptotically linear maps in ordered Banach spaces, J. Funct. Anal. 14 (1973), 162-171. MR 50:3019

3. H. Amann, Fixed point equations and nonlinear eigenvalue problems in ordered Banach spaces, SIAM Review 18 (1976), 620-709. MR 54:3519

4. N. P. Câc and J. A. Gatica, Fixed point theorems for mappings in ordered Banach spaces, J. Math. Anal. Appl. 71 (1979), 547-557. MR 80j:47071

5. J. Chmieliński, On the stability of the generalized orthogonality equation, In: Stability of Mappings of Hyers-Ulam Type (eds. Th. M. Rassias \& J. Tabor), Hadronic Press, Palm Harbour, Florida, 1994, pp. 31-57. MR 95j:39046

6. P. D. T. A. Elliott, Cauchy's functional equation in the mean, Advances in Math. 51 (1984), 253-257. MR 86d:39009

7. R. Ger, On functional inequalities stemming from stability questions, In: General Inequalities 6, (ed. W. Walter), Internat. Ser. Numer. Math., Vol. 103, Birkhäuser Verlag, Basel, 1992, pp. 227-240. MR 94b:39042

8. R. Ger and J. Sikorska, Stability of the orthogonal additivity, Bull. Polish Acad. Sci. Math. 43 (1995), 143-151. CMP 97:03

9. D. H. Hyers, On the stability of the linear functional equation, Proc. Nat. Acad. Sci. U.S.A. 27 (1941), 222-224. MR 2:315a

10. D. H. Hyers and Th. M. Rassias, Approximate homomorphisms, Aequat. Math. 44 (1992), 125-153. MR 93i:39007

11. G. Isac. Opérateurs asymptotiquement linéaires sur des espaces localement convexes, Colloq. Math. 46 (1982), 67-72. MR 84h:58014

12. G. Isac and Th. M. Rassias, On the Hyers-Ulam stability of $\psi$-additive mappings, J. Approx. Theory 72 (1993), 131-137. MR 94b:39043

13. G. Isac and Th. M. Rassias, Stability of $\psi$-additive mappings: Applications to nonlinear analysis, Intern. J. Math. \& Math. Sciences 19 (1996), 219-228. MR 96m:47114

14. M. A. Krasnoselskii, Positive Solutions of Operator Equations, Noordhoff, Groningen, (1964). MR 31:6107

15. Th. M. Rassias, On the stability of the linear mapping in Banach spaces, Proc. Amer. Math. Soc. 72 (1978), 297-300. MR 80d:47094

16. Th. M. Rassias and P. Šemrl, On the Hyers-Ulam stability of linear mappings, J. Math. Anal. Appl. 173 (1993), 325-338. MR 94d:39011

17. F. Skof, Sull' approssimazione delle applicazioni localmente $\delta$-additive, Atti. Accad. Sc. Torino 117 (1983), 377-389. MR 89a:39015

18. F. Skof, On the stability of functional equations on a restricted domain and a related topic, In: Stability of Mappings of Hyers-Ulam type (eds. Th. M. Rassias and J. Tabor), Hadronic Press, Palm Harbor, Florida, 1994, pp. 141-151. MR 96a:39035

Department of Mathematics, University of Southern California, los Angeles, CalIFORNIA 90089-1113

Department of Mathematics and Computer Science, Royal Military College of Canada, Kingston, Ontario, Canada K7K 5L0

Department of Mathematics, National Technical University of Athens, Zografou Campus, 15780 Athens, Greece 